\title{
SOLVABILITY OF THE POWER FLOW PROBLEM IN DC OVERHEAD WIRE CIRCUIT MODELING
}

\author{
Jakub ŠevČ́́́, Plzeň, LukÁš Adam, Praha, \\ JAN PŘIKRYL, VÁCLAV ŠMíDL, Plzeň
}

Received September 30, 2020. Published online July 16, 2021.

\begin{abstract}
Proper traffic simulation of electric vehicles, which draw energy from overhead wires, requires adequate modeling of traction infrastructure. Such vehicles include trains, trams or trolleybuses. Since the requested power demands depend on a traffic situation, the overhead wire DC electrical circuit is associated with a non-linear power flow problem. Although the Newton-Raphson method is well-known and widely accepted for seeking its solution, the existence of such a solution is not guaranteed. Particularly in situations where the vehicle power demands are too high (during acceleration), the solution of the studied problem may not exist. To deal with such cases, we introduce a numerical method which seeks maximal suppliable power demands for which the solution exists. This corresponds to introducing a scaling parameter to reduce the demanded power. The interpretation of the scaling parameter is the amount of energy which is absent in the system, and which needs to be provided by external sources such as on-board batteries. We propose an efficient two-stage algorithm to find the optimal scaling parameter and the resulting potentials in the overhead wire network. We perform a comparison with a naive approach and present a real-world simulation in the part of the Pilsen city in the Czech Republic. These simulations are performed in the traffic micro-simulator SUMO, a popular open-source traffic simulation platform.
\end{abstract}

Keywords: power flow problem; Newton-Raphson method; solvability; scaling parameter MSC 2020: 49Mxx, 65J15, 94C60, 90C30

This work was supported by the Ministry of Education, Youth and Sports of the Czech Republic under the project OP VVV Electrical Engineering Technologies with HighLevel of Embedded Intelligence, CZ.02.1.01/0.0/0.0/18_069/0009855, project OP VVV Research Center for Informatics, CZ.02.1.01/0.0/0.0/16_019/0000765, and by UWB Student Grant Project no. SGS-2021-021. 


\section{INTRODUCTION}

Electrification of transport belongs to one of the key targets of the automotive industry today. The electrification of public transport road vehicles in urban areas is feasible and well-used for decades employing trolleybuses or recently hybrid trolleybuses (i.e. dynamically charging e-buses with a battery pack on the board). A replacement of classic buses (with a combustion engine) with (hybrid) trolleybuses is, nonetheless, hardly possible without an appropriate adjustment and dimensioning of the necessary traction infrastructure. For this purpose, a simultaneous simulation of the power network and traffic conditions needs to be used to identify weaknesses of the proposed solution [15].

The trolleybus overhead wire network is typically a direct current (DC) electric circuit, where traction substations supply electric energy. The connected trolleybuses represent power loads. The steady state analysis of such circuits enables monitoring of voltage drops, undesirable over-currents, power losses and therefore effective dimensioning of suggested overhead wire networks in urban areas. In this manuscript, we consider the well-known DC power flow (PF) problem, where electric traction substations are modelled as constant voltage sources with voltages $V_{\bullet}$; the resistance of overhead wires is replaced by ideal resistor elements with the resistances $R_{\bullet}$ linearly dependent on the distances between nodes; trolleybuses are substituted by an electric current sources with power loads $P_{\bullet}$ proportional to source currents $I_{\bullet}$; and the corresponding connection nodes are associated with electric potentials $\varphi_{\bullet}$ (see a sample DC network in Figure 1).

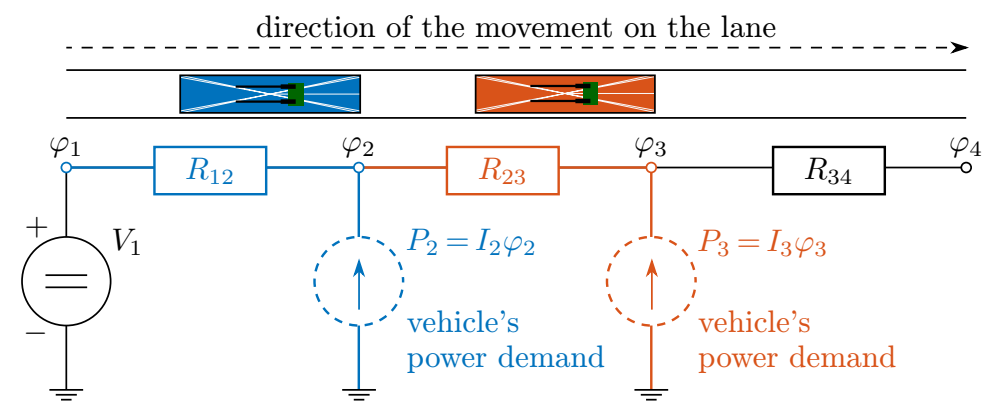

Figure 1. Simple DC network in ladder-form with moving trolleybuses.

Although there exists a rich literature on the alternating current (AC) power flow problem [16], papers on the DC PF problem were limited in the past. In the majority of cases, they dealt with the DC PF problem only from application point of view [14], [11], [18], [10] and without mentioning the solvability of the problem or uniqueness of the solution. Regardless, the majority of these works succeeded with utilizing Gauss- 
Seidel or Newton-Raphson methods to solve a well-defined system (i.e. system with well-defined operative conditions given by suitable values of variables) of DC PF non-linear equations. In recent years, the DC PF problem receives more attention in literature, since it is connected to low voltage DC grids, an appealing concept in the field of smart grids and microgrids.

Garces proves uniqueness [5] of the solution of the DC PF problem (using Banach fixed-point theorem), and even convergence of Gauss-Seidel and Newton-Raphson methods [6], [5], both under a set of reasonable and not much restricting assumptions but enforcing sufficiently low power demands. Further, Taylor's series expansion was used to linearize the DC PF problem in [13]. Taheri and Kekatos [16] proposed three various approaches to solve the DC PF problem assuming bounded power demands, and suggested a decision tree to select the proper method with guaranteed convergence. The DC PF problem was also reformulated as an optimization task [17], [7] and its solvability was discussed.

However, the existence of the solution of the DC PF problem generally heavily depends on the power demands [4]. Even for a primitive circuit (Figure 2), none, one, or two real-valued solutions of the DC PF problem may exist, depending on the value of the power demand. For the simple DC circuit on the left-hand side of Figure 2, the DC PF problem amounts to determining the unknown potential $\varphi_{2}$. The voltage $\varphi_{1}=V$ of the source, resistance $R$ of the resistor element, and requested power load $P$ are known. Using the Kirchhoff's law $R I_{2}=V-\varphi_{2}$, and the electric power equation $P=I_{2} \varphi_{2}$, we conclude that the unknown potential $\varphi_{2}$ is the solution of quadratic equation $\varphi_{2}^{2}-V \varphi_{2}+P R=0$. Therefore, there exists a critical value of power demand $P_{\text {crit }}=V^{2} / 4 R$ and the existence of the DC PF problem's solution depends on the ratio between the demanded power and the critical power value. If the demanded power is higher than the critical power value, the DC PF problem has no solution (see the graph on the right-hand side of Figure 2). In such situations, the demanded power cannot be supplied in real-world conditions due to physical restrictions.
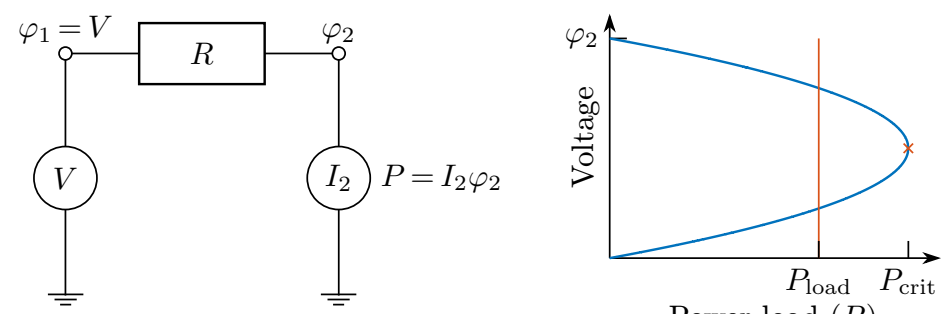

Power load $(P)$

Figure 2. Simple DC circuit (left) with unknown physical values emphasized with red color, and its solution (right). 
In this manuscript, we test the limitation of overhead wire infrastructure and simulate scenarios close to physical limits and even behind them. For this reason, a method solving the DC PF problem with large power loads is necessary. We introduce a scaling parameter that uniformly decreases the original demanded power values. We then propose a method to find a critical value of power demand where the overhead wire circuit is already solvable. Such decreased power values can be seen as the maximal suppliable power loads. This corresponds to finding a maximal value of the scaling parameter. We propose a two-stage strategy, where the first phase searches for the scaling parameter, while the second stage verifies the solvability of the overhead wire circuit. Since the scaling parameter is one-dimensional, this allows us to use a combination of a bisection method in the first stage and Newton-Raphson method in the second stage.

This paper is organized as follows. We revisit the mathematical formulation of the DC PF problem in Section 2, and we introduce a method for seeking maximal suppliable power demands to ensure the existence of the solution in Section 3. The theoretical analysis of the proposed approach is carried out in Section 4, where we show that our method is convergent under mild assumptions. A solution procedure employing introduced theoretical results and an efficient algorithm to find the solution with lower time requirements are proposed in Section 5. Finally, a numerical insight into the problem, a comparison with standard non-linear solver in Matlab, and a simulation of a city trolleybus network are presented in Section 6.

\section{Mathematical formulation}

The DC PF problem corresponds to finding unknown potentials of nodes and branch currents in an electric circuit with defined voltage and power loads/sources. There are various methods to formulate the corresponding set of equations. Since the fundamental electrical laws (Kirchhoff's circuit laws, Ohm's law, Power law) need to be always employed, the resulting formulations are naturally equivalent. We use modified nodal analysis [8] to form the system of equations for the electric circuit. Hence, given the specific application in overhead wire circuit modeling, the system (a connected electric circuit with voltage sources and power loads) can be described by three types of equations. Each equation describes currents going through a selected node with unknown potentials or assigns known voltage sources to adjacent nodes.

If node $i$ is not adjacent to any voltage source or power loads (i.e. the adjacent nodes are connected only through resistor elements), the Kirchhoff's current law states that the sum of currents flowing in and out of this node must be zero. Since 
current equals to voltage (difference of potentials) divided by resistance, this law amounts to

$$
\sum_{j \in N(i)} \frac{1}{R_{i j}}\left(\varphi_{i}-\varphi_{j}\right)=0, \quad i \in I,
$$

where $R_{i j}$ is the resistance between the $i$ th and $j$ th nodes, $\varphi_{i}$ is the potential of the $i$ th node, $N(i)$ is the set of adjacent nodes to the $i$ th nodes through resistor elements, and $I$ is an index set of nodes connected only by resistor elements.

If node $i$ is adjacent to a power load, we again use the Kirchoff's law. The lefthand side stays the same as in (2.1), but the right-hand side equals to the source current at the power load. Since this source current is $P_{i} / \varphi_{i}$, the equation reads

$$
\sum_{j \in N(i)} \frac{1}{R_{i j}}\left(\varphi_{i}-\varphi_{j}\right)=\frac{P_{i}}{\varphi_{i}}, \quad i \in J
$$

where $P_{i}$ is the demanded power by the power load adjacent to the $i$ th node and $J$ is an index set of nodes adjacent to any power load. Note that in the case of zero power demand $P_{i},(2.2)$ reduces to $(2.1)$ and $i$ is reassigned to set $I$.

The rest of nodes are connected to voltage sources, so their potentials equal to

$$
\varphi_{i}=U_{i}, \quad i \in I_{0}
$$

where $U_{i}$ is a known voltage level of voltage source at the $i$ th node and $I_{0}$ is an index set of nodes adjacent to any voltage source.

The DC PF problem then amounts to finding the unknown potentials $\varphi_{i}, i \in$ $\left\{I, J, I_{0}\right\}$. If we solve equations (2.1)-(2.3) for unknown potentials, currents through the circuit can be found by Ohm's and Power laws.

System (2.1)-(2.3) can be rewritten in a compact form introducing a square matrix $A$ and a vector $\boldsymbol{b}(\boldsymbol{\varphi})$ :

$$
A \varphi=b(\varphi) \equiv\left[\begin{array}{c}
0 \\
\frac{P}{\varphi_{J}} \\
U
\end{array}\right]
$$

where

$$
A_{i, j}= \begin{cases}\sum_{k \in N(i)} \frac{1}{R_{i k}} & \text { if } i=j \wedge i \in I \cup J, \\ -\frac{1}{R_{i j}} & \text { if } i \neq j \wedge i \in I \cup J \wedge j \in N(i), \\ 1 & \text { if } i=j \wedge i \in I_{0}, \\ 0 & \text { otherwise. }\end{cases}
$$


Here, $\boldsymbol{\varphi}$ is an ordered vector of unknown potentials $\varphi_{i}, i \in\left\{I, J, I_{0}\right\}, \mathbf{0}$ is a zero vector, $\boldsymbol{P}$ is a vector of power demands with non-zero components $P_{i}, i \in J, \boldsymbol{\varphi}_{J}$ is a vector with components $\varphi_{i}, i \in J, \boldsymbol{P} / \boldsymbol{\varphi}_{J}$ is the element-wise division and the vector $\boldsymbol{U}$ consists of components $U_{i}, i \in I_{0}$.

\section{ReduCtion OF POWER DEMANDS TO ENSURE SOLVABILITY}

To find a solution of equation (2.4), we can employ numerical methods as the Gauss-Seidel method or Newton-Raphson method. However, as it has been discussed in Section 1, the existence of the solution is not guaranteed in all situations. Especially if the power demands are too high, the solution of the problem does not exist. In such a situation, the power demands of trolleybuses cannot be fully satisfied due to physical limits or due to over-current protections of traction substation.

To find the maximal suppliable values of power and to guarantee the existence of a solution, we adjust (2.2) by introducing a vector $\boldsymbol{\alpha}$ of scaling parameters $\alpha_{i}$ by

$$
\sum_{j \in N(i)} \frac{1}{R_{i j}}\left(\varphi_{i}-\varphi_{j}\right)=\frac{\alpha_{i} P_{i}}{\varphi_{i}}, \quad i \in J .
$$

Equations (2.1) and (2.3) are without any change. To simplify matters, parameter vector $\boldsymbol{\alpha}$ is considered as all-ones vector multiplied by a scalar value, i.e. $\boldsymbol{\alpha}=\alpha \mathbf{1}$. While this formulation does not fully correspond to the physical equilibrium of the system, the scalar approximation makes the system computationally tractable. Note that $\alpha=1$ corresponds to the original problem and $\alpha=0$ to the situation when no demanded power is supplied. Again, the problem can be reformulated into the compact form similar to $(2.4)$,

$$
f(\boldsymbol{\varphi}(\alpha), \alpha):=A \boldsymbol{\varphi}(\alpha)-\boldsymbol{b}(\boldsymbol{\varphi}(\alpha), \alpha):=A \boldsymbol{\varphi}(\alpha)-\left[\begin{array}{c}
\mathbf{0} \\
\frac{\alpha \boldsymbol{P}}{\boldsymbol{\varphi}(\alpha)_{J}} \\
\boldsymbol{U}
\end{array}\right]=0,
$$

where we denote the dependency of $\varphi$ on the scalar parameter $\alpha$ by $\varphi(\alpha)$.

The value of $\alpha$ can be seen as an overload rate of the investigated electric circuit, or in other words, as a power demand satisfaction rate. If equation (3.2) has the solution for $\alpha=1$ (i.e. the original problem), the investigated circuit is not overloaded and all power loads are fully supplied by the circuit. For $\alpha=0$, i.e. the power demands are completely disregarded, (3.2) has always a trivial solution with zero currents and with nominal voltage of traction substation at all nodes in the circuit.

The idea of the proposed method is to find some $\alpha_{0} \in[0,1]$ such that (3.2) has a solution for $\alpha \in\left[0, \alpha_{0}\right]$ and does not have a solution for $\alpha \in\left(\alpha_{0}, 1\right]$. Since the 
scaling parameter evenly decreases values of the demanded power, $\alpha_{0}$ can be also defined as a maximal $\alpha \in[0,1]$, for which (3.2) has a solution. The optimal $\alpha_{0}$ then determines the maximal power threshold which can be provided by the circuit and determines the circuit overload rate in some sense. Finding such $\alpha_{0}$ corresponds to an optimization task

$$
\alpha_{0}=\arg \max _{\alpha, \boldsymbol{\varphi}} \alpha \quad \text { subject to } A \boldsymbol{\varphi}(\alpha)=\boldsymbol{b}(\boldsymbol{\varphi}(\alpha), \alpha), \quad \alpha \in[0,1] .
$$

The optimal $\alpha_{0}$ smaller than one gives us the information that the overhead wire is overloaded and determines the rate of this overload.

\section{Theoretical AnALYSiS}

The theoretical analysis justifying the proposed approach needs to make four assumptions. The first one reads:

(A1) If system (2.1), (3.1), (2.3) has a solution $\boldsymbol{\varphi ( 1 )}$ for some $\boldsymbol{P}$, then it has a solution for all $\alpha \boldsymbol{P}$ with $\alpha \in[0,1]$.

This assumption says that if the system can be satisfied for a power demand $\boldsymbol{P}$, it can be satisfied for all smaller power demands. This assumption also has a dual interpretation. Consider the solution $\varphi(1)$ of (2.1), (3.1), (2.3) with $\alpha=1$, fix an arbitrary $\alpha \in[0,1]$ and define $\boldsymbol{\theta}(\alpha)=\sqrt{\alpha} \boldsymbol{\varphi}(1)$. Then $\boldsymbol{\theta}$ solves (2.1) and (3.1) and it satisfies (2.3) with the reduced source voltage $\hat{U}_{i}=\sqrt{\alpha} U_{i} \leqslant U_{i}$. Since the solvability problem arises due to the inability to provide enough energy for the requested power demand (Figure 2), we conjecture that a power demand that can be satisfied by $\sqrt{\alpha} U_{i}$ can be also satisfied by full voltage $U_{i}$.

The second assumption requires topology of the network:

(A2) The network is connected and there exists at least one voltage source in the network.

For the last two assumptions, consider the set of equations

$$
f(\boldsymbol{\varphi}(\boldsymbol{\alpha}), \boldsymbol{\alpha}):=A \varphi(\boldsymbol{\alpha})-\boldsymbol{b}(\boldsymbol{\varphi}(\boldsymbol{\alpha}), \boldsymbol{\alpha}):=A \varphi(\boldsymbol{\alpha})-\left[\begin{array}{c}
\mathbf{0} \\
\frac{\boldsymbol{\alpha} \circ \boldsymbol{P}}{\boldsymbol{\varphi}(\boldsymbol{\alpha})_{J}} \\
\boldsymbol{U}
\end{array}\right]=0
$$

to allow reducing the power demands in an unequal manner. The notation $\boldsymbol{\alpha} \circ \boldsymbol{P}$ denotes the Hadamard (component-wise) product of two vectors, and the fraction of two vectors is considered also in component-wise manner. Define the solution mapping of (4.1) by

$$
S(\boldsymbol{\alpha}):=\{\boldsymbol{\varphi} \mid \boldsymbol{\varphi} \text { solves (4.1) for } \boldsymbol{\alpha}\}
$$


and its domain by

$$
\mathcal{D}:=\{\boldsymbol{\alpha} \mid S(\boldsymbol{\alpha}) \text { is nonempty }\} \cap[0,1]^{|\boldsymbol{\alpha}|} .
$$

Then we impose the third assumption:

(A3) There exists some $M$ such that $\|\boldsymbol{y}\| \leqslant M$ for all $\boldsymbol{\alpha} \in \mathcal{D}$ and $\boldsymbol{y} \in S(\boldsymbol{\alpha})$.

This says that the electric potential cannot be infinite. The last assumption states:

(A4) The solution mapping $S$ has continuously differentiable single-valued localization around each point from the interior of $\mathcal{D}$.

This assumption is also natural and makes sense from physical point of view. We explain its meaning at the simple example from Figure 2 with $P=P_{\text {crit }}$. This figure depicts the scaled graph of the solution mapping $S$. Whenever $\alpha \in[0,1)$, there are two solutions to the system. Therefore, $S(\alpha)$ contains two points and we have to work with single-valued localizations, which select either the upper or the lower branch in Figure 2. These localizations are continuously differentiable, which is precisely what Assumption (A4) states.

To summarize, all assumptions (A1)-(A4) are well justified and always satisfied in real-world electrical systems. We start with the following two lemmas.

Lemma 4.1. Let Assumption (A2) be satisfied. If $\varphi_{i}$ are fixed for all $i \in J \cup I_{0}$, then (2.1) has a unique solution $\varphi_{i}$ with $i \in I$.

P r o of. We define $A_{I I}$ as the restriction of $A$ to rows and columns $I$. Similarly, we define $A_{I I_{0}}$. To prove the statement, we need to show that $A_{I I}$ is a non-singular matrix. We define

$$
B=\left(\begin{array}{cc}
A_{I I} & A_{I I_{0}} \\
0 & E
\end{array}\right)
$$

to be the restriction of $A$ to rows and columns $I \cup I_{0}$. Here, $E$ denotes the identity matrix.

We will use some results from graph theory. We recall that the matrix $B$ is strictly diagonally dominant if

$$
\left|b_{i i}\right|>\sum_{j \neq i}\left|b_{i j}\right|
$$

for each $i$. The matrix $B$ is weakly diagonally dominant if the inequalities are replaced by $\geqslant$, and weakly chained diagonally dominant if it is weakly diagonally dominant and each node $i$ is connected via a path to a strictly diagonally dominant node. Due to the construction of the matrix $A$, all nodes of $B$ are weakly diagonally dominant, and all nodes corresponding to voltage sources are strictly diagonally dominant. Then Assumption (A2) implies that $B$ is weakly chained diagonally dominant. Since every such matrix is non-singular, $B$ is non-singular and therefore, $A_{I I}$ is non-singular as well. This finishes the proof of the lemma. 
Lemma 4.2. Let Assumption (A3) be satisfied. Then the domain $\mathcal{D}$ is a closed set.

P r o o f. Consider any sequence $\boldsymbol{\alpha}_{k} \rightarrow \boldsymbol{\alpha}$ such that $\boldsymbol{\alpha}_{k} \in \mathcal{D}$ for all $k$. We need to show that $\boldsymbol{\alpha} \in \mathcal{D}$. There are some $\boldsymbol{\varphi}_{k} \in S\left(\boldsymbol{\alpha}_{k}\right)$. Due to Assumption (A3), they are bounded and we may select a convergent subsequence, denoted without loss of generality by the same indices, $\varphi_{k} \rightarrow \boldsymbol{\varphi}$. Since $f$ from (4.1) is continuous with respect to both variables, we have $\boldsymbol{\varphi} \in S(\boldsymbol{\alpha})$, which implies $\boldsymbol{\alpha} \in \mathcal{D}$.

Now we are able to prove the following theorem.

Theorem 4.1. Let Assumptions (A1)-(A4) be satisfied. Then there are two possibilities:

$\triangleright$ System (3.2) has a solution for all $\alpha \in[0,1]$.

$\triangleright$ System (3.2) has a solution for all $\alpha \in\left[0, \alpha_{0}\right]$ but no solution for any $\alpha \in\left(\alpha_{0}, 1\right]$ for some $\alpha_{0} \in[0,1)$. In such a case, the Jacobian matrix $\nabla_{\varphi} f(\varphi(\alpha), \alpha)$ is non-singular for all $\alpha \in\left[0, \alpha_{0}\right)$ but $\nabla_{\varphi} f\left(\varphi\left(\alpha_{0}\right), \alpha_{0}\right)$ is singular.

P r o o f. We realize that due to Assumption (A1), either system (3.2) has a solution for all $\alpha \in[0,1]$ or there is some $\alpha_{0} \in[0,1)$ such that the system has a solution on $\left[0, \alpha_{0}\right)$ and no solution on $\left(\alpha_{0}, 1\right]$. Lemma 4.2 and the existence of the trivial solution for $\alpha=0$ imply that (3.2) has a solution even for $\alpha_{0}$ in the second case.

It remains to show in which cases the Jacobians are non-singular. Fix any $\alpha \in$ $\left[0, \alpha_{0}\right]$ and define its vector version $\boldsymbol{\alpha}=\alpha \mathbf{1}$. We write (4.1) in a more compact form

$$
\left(\begin{array}{ccc}
A_{I I} & A_{I J} & A_{I I_{0}} \\
A_{J I} & A_{J J} & A_{J I_{0}} \\
0 & 0 & E
\end{array}\right)\left(\begin{array}{c}
\varphi_{I} \\
\boldsymbol{\varphi}_{J} \\
\boldsymbol{\varphi}_{I_{0}}
\end{array}\right)=\left(\begin{array}{c}
\mathbf{0} \\
\frac{1}{\boldsymbol{\varphi}_{J}}(\boldsymbol{\alpha} \circ \boldsymbol{P}) \\
\boldsymbol{U}
\end{array}\right)
$$

where $E$ stands for the identity matrix. The matrix on the left-hand side does not depend on the potentials $\varphi$. Due to Lemma 4.1, the square matrix $A_{I I}$ is non-singular and we have

$$
\boldsymbol{\varphi}_{I}=A_{I I}^{-1}\left(-A_{I J} \varphi_{J}-A_{I I_{0}} \varphi_{I_{0}}\right) .
$$

Plugging this back to (4.2) yields

$$
A_{J J} \boldsymbol{\varphi}_{J}+A_{J I} A_{I I}^{-1}\left(-A_{I J} \boldsymbol{\varphi}_{J}-A_{I I_{0}} \boldsymbol{\varphi}_{I_{0}}\right)+A_{J I_{0}} \boldsymbol{\varphi}_{I_{0}}=\frac{1}{\boldsymbol{\varphi}_{J}}(\boldsymbol{\alpha} \circ \boldsymbol{P})
$$

and in a simpler form

$$
g\left(\boldsymbol{\varphi}_{J}\right):=\boldsymbol{\varphi}_{J} \circ\left(A_{J J}-A_{J I} A_{I I}^{-1} A_{I J}\right) \boldsymbol{\varphi}_{J}-\boldsymbol{\varphi}_{J} \circ\left(A_{J I} A_{I I}^{-1} A_{I I_{0}} \boldsymbol{U}-A_{J I_{0}} \boldsymbol{U}\right)=\boldsymbol{\alpha} \circ \boldsymbol{P}
$$


Now we perturb the right-hand side of (4.3) by some $\boldsymbol{r}$ to get

$$
\boldsymbol{\varphi}_{J} \circ\left(A_{J J}-A_{J I} A_{I I}^{-1} A_{I J}\right) \boldsymbol{\varphi}_{J}-\boldsymbol{\varphi}_{J} \circ\left(A_{J I} A_{I I}^{-1} A_{I I_{0}} \boldsymbol{U}-A_{J I_{0}} \boldsymbol{U}\right)=\boldsymbol{\alpha} \circ \boldsymbol{P}+\boldsymbol{r}
$$

and define $\widetilde{S}: \boldsymbol{r} \mapsto \varphi_{J}$ as a solution mapping of (4.4). We consider $\boldsymbol{\alpha}$ as a fixed parameter and perturb only $\boldsymbol{r}$. Equality (4.4) amounts to

$$
\boldsymbol{\varphi}_{J} \circ\left(A_{J J}-A_{J I} A_{I I}^{-1} A_{I J}\right) \boldsymbol{\varphi}_{J}-\boldsymbol{\varphi}_{J} \circ\left(A_{J I} A_{I I}^{-1} A_{I I_{0}} \boldsymbol{U}-A_{J I_{0}} \boldsymbol{U}\right)=\left(\boldsymbol{\alpha}+\frac{\boldsymbol{r}}{\boldsymbol{P}}\right) \circ \boldsymbol{P} .
$$

This, due to the same reasons as above, is equivalent to $f(\boldsymbol{\varphi}(\widetilde{\boldsymbol{\alpha}}), \widetilde{\boldsymbol{\alpha}})=0$ with $\widetilde{\alpha}_{i}=\alpha_{i}+r_{i} / P_{i}, i \in J$. This implies $\widetilde{S}(\boldsymbol{r})=S(\boldsymbol{\alpha}+\boldsymbol{r} / \boldsymbol{P})$ and therefore, local properties of $\widetilde{S}$ around $\mathbf{0}$ correspond to those of $S$ around $\boldsymbol{\alpha}$. In particular, the existence of a continuously differentiable single-valued localization of $\widetilde{S}$ around $\mathbf{0}$ is equivalent to the existence of a continuously differentiable single-valued localization of $S$ around $\boldsymbol{\alpha}$. Due to [3], Theorem 1C.3, the existence of the former happens if and only if $\nabla_{\varphi} g\left(\boldsymbol{\varphi}_{J}(\boldsymbol{\alpha})\right)$, defined in (4.3), is non-singular. Combining these two facts we obtain that $S$ has a continuously differentiable single-valued localization at $\boldsymbol{\alpha}$ if and only if $\nabla_{\varphi} g\left(\boldsymbol{\varphi}_{J}(\boldsymbol{\alpha})\right)$ is non-singular. Assumption (A4) ensures that this holds true whenever $\boldsymbol{\alpha}=\alpha \mathbf{1}$ and $\alpha \in\left[0, \alpha_{0}\right)$. At the same time, it cannot happen for $\boldsymbol{\alpha}=\alpha_{0} \mathbf{1}$, because this point is at the boundary of $\mathcal{D}$. This whole paragraph implies that $\nabla_{\varphi} g\left(\boldsymbol{\varphi}_{J}(\boldsymbol{\alpha})\right)$ is non-singular for all $\alpha \in\left[0, \alpha_{0}\right)$ but $\nabla_{\varphi} g\left(\boldsymbol{\varphi}_{J}\left(\boldsymbol{\alpha}_{0}\right)\right)$ is singular.

The non-singularity of $\nabla_{\varphi} g\left(\varphi_{J}\right)$ is equivalent to the non-singularity of the Jacobian of

$$
\hat{g}\left(\boldsymbol{\varphi}_{J}\right):=\left(A_{J J}-A_{J I} A_{I I}^{-1} A_{I J}\right) \boldsymbol{\varphi}_{J}-\left(A_{J I} A_{I I}^{-1} A_{I I_{0}} \boldsymbol{U}-A_{J I_{0}} \boldsymbol{U}\right)-\frac{1}{\boldsymbol{\varphi}_{J}}(\boldsymbol{\alpha} \circ \boldsymbol{P}) .
$$

We have

$$
\nabla_{\varphi} \hat{g}\left(\boldsymbol{\varphi}_{J}(\boldsymbol{\alpha})\right)=A_{J J}-A_{J I} A_{I I}^{-1} A_{I J}+\operatorname{diag}\left(\frac{1}{\boldsymbol{\varphi}_{J}^{2}}(\boldsymbol{\alpha} \circ \boldsymbol{P})\right),
$$

where $\operatorname{diag}(\cdot)$ makes a diagonal matrix from a vector and $\varphi_{J}^{2}$ is understood component-wise. Define now the function from (4.2) by

$$
\tilde{g}(\boldsymbol{\varphi}):=\left(\begin{array}{ccc}
A_{I I} & A_{I J} & A_{I I_{0}} \\
A_{J I} & A_{J J} & A_{J I_{0}} \\
0 & 0 & E
\end{array}\right)\left(\begin{array}{c}
\varphi_{I} \\
\boldsymbol{\varphi}_{J} \\
\boldsymbol{\varphi}_{I_{0}}
\end{array}\right)-\left(\begin{array}{c}
\mathbf{0} \\
\frac{1}{\boldsymbol{\varphi}_{J}}(\boldsymbol{\alpha} \circ \boldsymbol{P}) \\
\boldsymbol{U}
\end{array}\right) .
$$

Then

$$
\nabla_{\varphi} \tilde{g}(\boldsymbol{\varphi}(\boldsymbol{\alpha}))=\left(\begin{array}{ccc}
A_{I I} & A_{I J} & A_{I I_{0}} \\
A_{J I} & A_{J J}+\operatorname{diag}\left(\frac{1}{\boldsymbol{\varphi}_{J}^{2}}(\boldsymbol{\alpha} \circ \boldsymbol{P})\right) & A_{J I_{0}} \\
0 & 0 & E
\end{array}\right)
$$


and therefore,

$$
\begin{aligned}
\operatorname{det} \nabla_{\varphi} \tilde{g}(\boldsymbol{\varphi}(\boldsymbol{\alpha})) & =\operatorname{det}\left(\begin{array}{cc}
A_{I I} & A_{I J} \\
A_{J I} & A_{J J}+\operatorname{diag}\left(\frac{1}{\boldsymbol{\varphi}_{J}^{2}}(\boldsymbol{\alpha} \circ \boldsymbol{P})\right)
\end{array}\right) \\
& =\operatorname{det} A_{I I} \operatorname{det}\left(A_{J J}+\operatorname{diag}\left(\frac{1}{\boldsymbol{\varphi}_{J}^{2}}(\boldsymbol{\alpha} \circ \boldsymbol{P})\right)-A_{J I} A_{I I}^{-1} A_{I J}\right) \\
& =\operatorname{det} A_{I I} \operatorname{det} \nabla_{\varphi} \hat{g}\left(\boldsymbol{\varphi}_{J}(\boldsymbol{\alpha})\right),
\end{aligned}
$$

where the second equality follows from the theory of Schur's complement, and the third equality from (4.5). Lemma 4.1 says that $\operatorname{det} A_{I I} \neq 0$ and therefore $\operatorname{det} \nabla_{\varphi} \tilde{g}(\boldsymbol{\varphi}(\boldsymbol{\alpha})) \neq 0$ if and only if $\operatorname{det} \nabla_{\varphi} \hat{g}\left(\boldsymbol{\varphi}_{J}(\boldsymbol{\alpha})\right) \neq 0$. Combining this with the other equivalence of non-singularity of Jacobians from the beginning of this paragraph, yields that $\nabla_{\varphi} \tilde{g}(\boldsymbol{\varphi}(\boldsymbol{\alpha}))$ is non-singular if and only if $\nabla_{\varphi} g\left(\boldsymbol{\varphi}_{J}(\boldsymbol{\alpha})\right)$ is non-singular. Recalling the notation $\boldsymbol{\alpha}=\alpha \mathbf{1}$, we realize that $f(\boldsymbol{\varphi}(\alpha), \alpha)=\tilde{g}(\boldsymbol{\varphi}(\boldsymbol{\alpha}))$. Since we have already shown that $\nabla_{\varphi} g\left(\boldsymbol{\varphi}_{J}(\boldsymbol{\alpha})\right)$ is non-singular for all $\alpha \in\left[0, \alpha_{0}\right)$ but $\nabla_{\varphi} g\left(\boldsymbol{\varphi}_{J}\left(\boldsymbol{\alpha}_{0}\right)\right)$ is singular, this implies the theorem statement.

Assuming (A1)-(A4), Theorem 4.1 ensures that the optimization task (3.3) has nice properties which we will utilize to suggest a solution algorithm in the next section and to further investigate and numerically demonstrate it in Section 6 .

Theorem 4.1 is closely connected with the homotopy method [1], which solves a complicated system by linearly interpolating it with a simple system. In our case, the simple system corresponds to $\alpha=0$, the difficult system to $\alpha=\alpha_{0}$ and the linear interpolation is governed by increasing $\alpha$.

A related result to our Theorem 4.1 appeared in [2], Theorem 3, where the authors showed that the interpolation scheme results in a connected path for which a solution exists for all $\alpha \in\left[0, \alpha_{0}\right]$. This is our Assumption (A1). On the other hand, their Assumption (G4) is precisely our conclusion about the non-singularity of $\nabla_{\varphi} f(\varphi(\alpha), \alpha)$. In other words, these two results are inverse to each other.

\section{Solution Procedure}

In this section, we propose a method with simple implementation and low computation requirements. Our method is based on Theorem 4.1, which states that there is some $\alpha_{0}$ such that system (3.2) is solvable for all $\alpha \in\left[0, \alpha_{0}\right]$ but not solvable for any larger $\alpha$. Therefore, we start with $\widetilde{\alpha}=0$ and incrementally increase the value of $\widetilde{\alpha}$ by some constant gain $\Delta \alpha$. After each update of the scaling parameter, the 
Newton-Raphson method is used to solve (3.2), with the $k$ th iteration evaluated as

$$
\begin{aligned}
\boldsymbol{\varphi}_{\mathrm{k}+1} & =\boldsymbol{\varphi}_{\mathrm{k}}-\left(\nabla_{\boldsymbol{\varphi}} f\left(\boldsymbol{\varphi}_{\mathrm{k}}, \widetilde{\alpha}\right)\right)^{-1} f\left(\boldsymbol{\varphi}_{k}, \widetilde{\alpha}\right) \\
& =\boldsymbol{\varphi}_{\mathrm{k}}-\left(A-\nabla_{\boldsymbol{\varphi}} \boldsymbol{b}\left(\boldsymbol{\varphi}_{\mathrm{k}}, \widetilde{\alpha}\right)\right)^{-1}\left(A \boldsymbol{\varphi}_{\mathrm{k}}-\boldsymbol{b}\left(\boldsymbol{\varphi}_{\mathrm{k}}, \widetilde{\alpha}\right)\right)
\end{aligned}
$$

Theorem 4.1 also states that $\nabla_{\varphi} f\left(\varphi_{\mathrm{k}}, \widetilde{\alpha}\right)$ converges to a singular matrix as $\widetilde{\alpha} \rightarrow \alpha_{0}$. Therefore, if we observe that the determinant of this matrix (which is the same as the one in (5.1)) goes to zero, we imply that we are close to the optimal scaling parameter $\alpha_{0}$. If this happens, the previous value of $\widetilde{\alpha}$ is declared as the optimal value with tolerance equal to $\Delta \alpha$. This procedure is summarized in Algorithm 1.

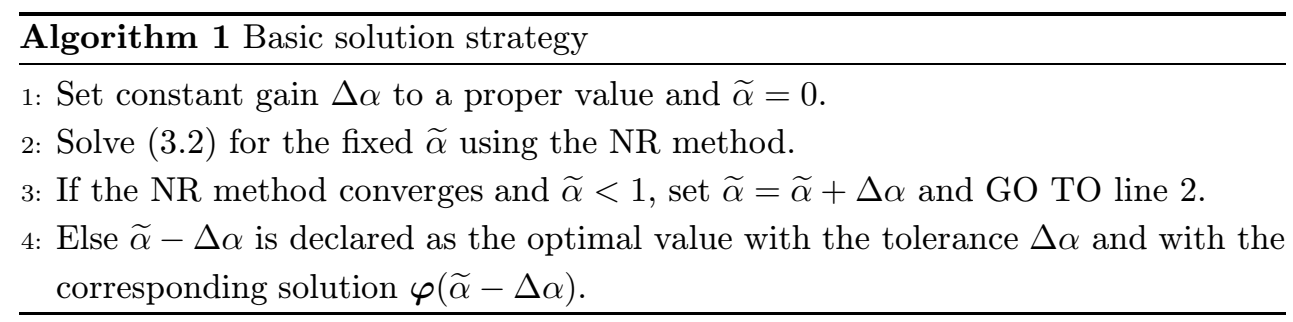

The suggested procedure is convergent under the assumptions of Theorem 4.1. However, it has a significant drawback in the case when the original DC PF problem (2.4) is solvable (i.e. there exists solution of (3.2) for $\alpha=1$ ). Then, all discretized values of $\alpha$ need to be passed before the optimal $\alpha_{0}=1$ is encountered. For this reason, we propose an efficient solution procedure in the next subsection.

5.1. Efficient solution algorithm. This efficient procedure replaces the incremental increase of $\widetilde{\alpha}$ by a variant of a bisection method. The complete proposed pseudo-code is shown in Algorithm 2 and its $\mathrm{C}++$ implementation can be found on official Eclipse SUMO GitHub repository ${ }^{1}$.

The while loop determines the optimal value of the scaling parameter. It starts with $\widetilde{\alpha}=1$ and makes use of the NR method to solve (3.2). The NR updates are inside the for loop. If the NR method succeeds (within a tolerance $\Delta_{\text {con }}$ ), the lower bound $\widehat{\alpha}$ is updated. In the opposite case the buffer of failed candidate values $S_{\alpha}$ is extended by the current scaling parameter $\widetilde{\alpha}$. If the buffer is empty (which happens if the NR method converged for $\widetilde{\alpha}=1$ ), we found a solution of (3.2) and the algorithm terminates.

If the buffer is not empty, it means that there are some scaling parameters for which the solution does not exist. In most cases, we determine the new $\widetilde{\alpha}$ as an

\footnotetext{
${ }^{1}$ https://github.com/eclipse/sumo/blob/master/src/utils/traction_wire/ Circuit.cpp
} 


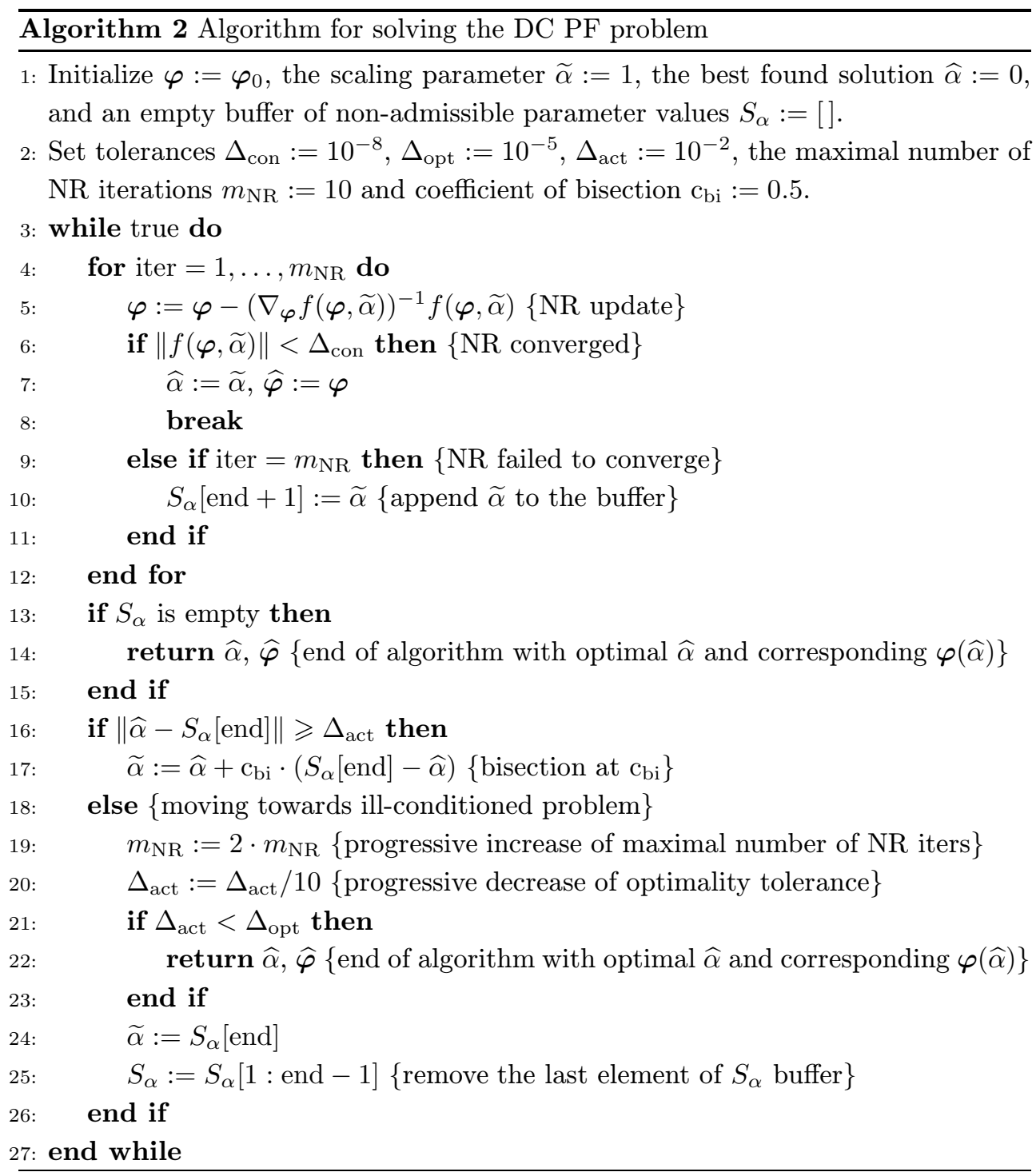

interpolation between $\widehat{\alpha}$ and the smallest value of the buffer $S_{\alpha}$. However, if these two values are close to each other (measured by the prescribed tolerance $\Delta_{\text {act }}$ ), the NR may require more iterations to converge, since the problem is ill-conditioned due to Theorem 4.1. In such a case, we double the allowed number of iterations for the NR algorithm. This is depicted in the last few lines of Algorithm 2.

Since the convergence of the NR method also depends on the initial estimate, we initialize it by assuming constant term $\boldsymbol{b}(U, 1)$ in $(3.2)$, where $U$ is the known nominal 
voltage of the traction substation, and then by solving the linear equation $A \varphi_{0}=$ $\boldsymbol{b}(U, 1)$ for $\varphi_{0}$. In the numerical implementation, we also incorporate current limits of traction substations (over-current protection) and voltage limits of the network in a simple way. However, for the sake of simplicity we will ignore them here.

The proposed algorithm is simple to implement and its computation requirements are lower than those required by classical approaches such as interior-point methods. Furthermore, the initialization of $\widetilde{\alpha}=1$ is strongly beneficial as the proposed algorithm solves the original unlimited DC PF problem within one iteration. Then the solution is found quickly without any additional computational requirements.

\section{Simulation AND numerical RESUlts}

For numerical validation, we consider toy examples as well as a simulation of real-world traffic.

6.1. Toy examples. The two toy test cases of the DC PF problem are motivated by a real trolleybus network. The first test case (Figure 3a) contains four vehicles with defined power demands $(260 \mathrm{~kW}, 20 \mathrm{~kW}, 30 \mathrm{~kW}$ and $-5 \mathrm{~kW}$ due to regenerative braking) running under overhead wire section connected to the traction substation represented by voltage source of $600 \mathrm{~V}$ using one connection point. The second test case (Figure 3b) contains ten vehicles with uniform power demands of $250 \mathrm{~kW}$ and two connection points to the traction substation on the voltage level $600 \mathrm{~V}$. The mean distance between adjacent (neighboring) vehicles is in the order of hundreds of metres in both test cases; this corresponds to the mean resistance of $0.023 \Omega$ to $0.23 \Omega$ of conductor wire between voltage nodes.

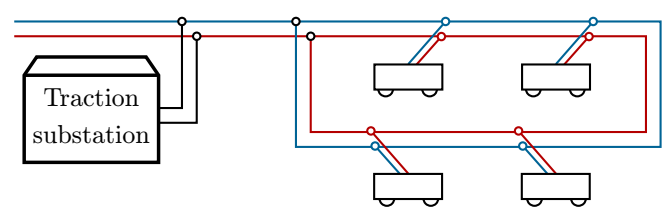

(a)

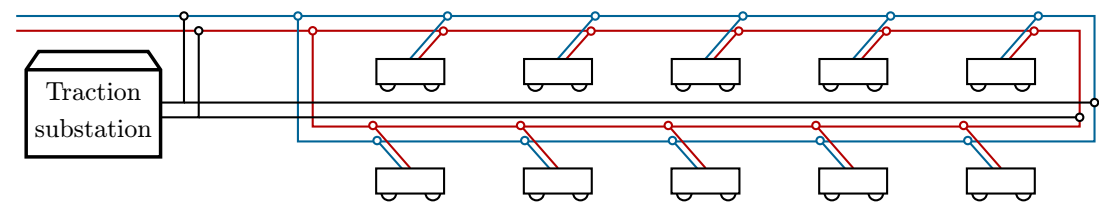

(b)

Figure 3. The scheme of the two toy test cases used for numerical analysis. 

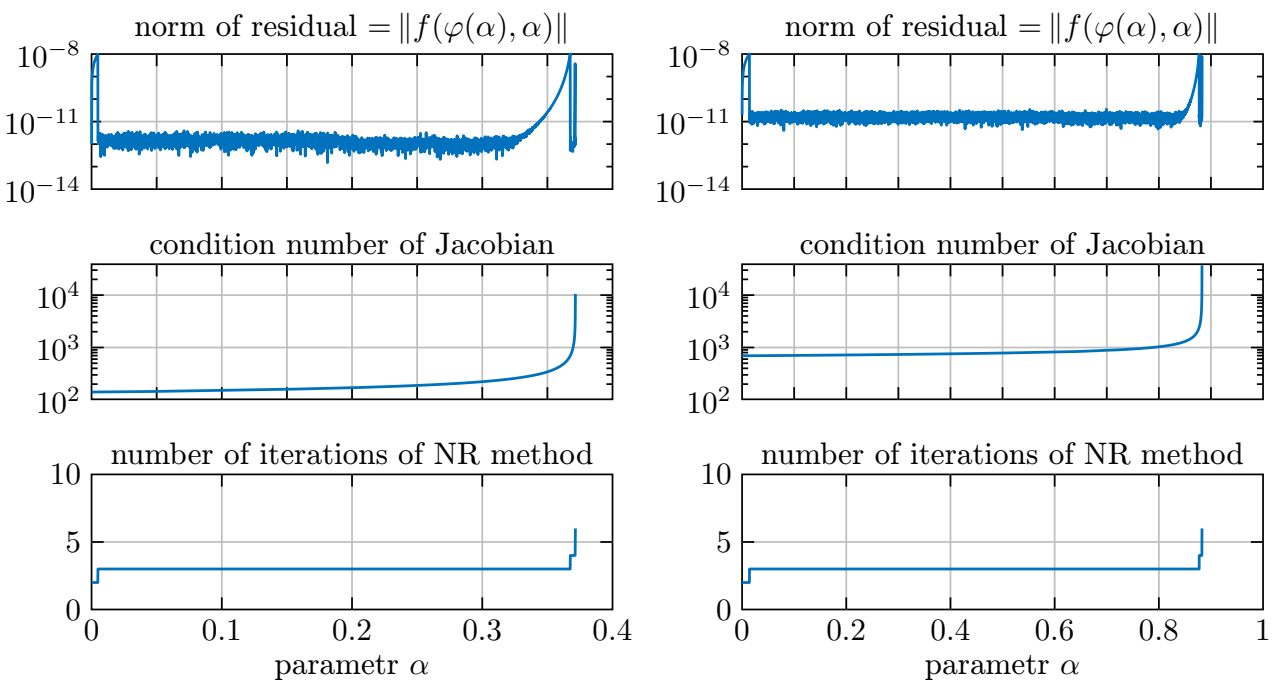

Figure 4. Dependency of the condition number of Jacobian on the proposed scaling parameter for the first toy case (left) and the second toy case (right).

Figure 4 empirically confirms the statements of Lemma 4.2 and Theorem 4.1. The left and right column of the figure show results for both toy cases introduced above. The top row shows the residuum of the solution which is always smaller than the threshold $\Delta_{\text {con }}=10^{-8}$. The middle row shows the condition number of the Jacobian $\nabla_{\varphi} f(\varphi(\alpha), \alpha)$ and the bottom row the number of iterations needed by the NewtonRaphson method. We see that this number is small but it is rising up as the condition number of Jacobian increases. The steep increase of the value of the condition number of Jacobian $\nabla_{\varphi} f(\varphi(\alpha), \alpha)$ in the middle row is apparently close to the critical value $\alpha_{0}$. It exactly corresponds to the theoretical conclusion of Theorem 4.1 stating that the interval of non-singularity of Jacobian matrix $\nabla_{\varphi} f(\varphi(\alpha), \alpha)$ is bounded by the critical value $\alpha_{0}$. Finally, we can see that the results of Lemma 4.2 are also numerically confirmed as the solution exists only on some interval.

Let us now compare Matlab implementation of our Algorithm 2 with the standard Matlab built-in function fmincon for solving constrained non-linear optimization problems using the interior-point method by default. The optimal scaling parameters $\alpha_{0}=0.3715$ for the first test case and $\alpha_{0}=0.8849$ for the second test case were successfully found both by Algorithm 2 and by fmincon. The time requirements are compared in Table 1. Calculations were performed in Matlab on Intel Core i5 processor and the time requirements are the average over 1000 evaluations. Our Algorithm 2 gives the solution about one order of magnitude faster than fmincon. If we provide user-defined gradient to fmincon, the time requirements are still approximately six times higher than for Algorithm 2. 


\begin{tabular}{ccccccc}
\hline & \multicolumn{3}{c}{ Test case 1 } & \multicolumn{3}{c}{ Test case 2 } \\
Algorithms & mean $t$ & $\max t$ & $\min t$ & $\operatorname{mean} t$ & $\max t$ & $\min t$ \\
& {$[\mathrm{~s}]$} & {$[\mathrm{s}]$} & {$[\mathrm{s}]$} & {$[\mathrm{s}]$} & {$[\mathrm{s}]$} & {$[\mathrm{s}]$} \\
\hline fmincon & 0.0266 & 0.0484 & 0.0221 & 0.0403 & 0.0913 & 0.0355 \\
fmincon with gradients & 0.0217 & 0.0412 & 0.0177 & 0.0261 & 0.0605 & 0.0219 \\
proposed Algorithm 2 & 0.0032 & 0.0059 & 0.0027 & 0.0042 & 0.0096 & 0.0040 \\
\hline
\end{tabular}

Table 1. Comparison of mean, maximal and minimal time requirements of proposed Algorithm 2 and Matlab's fmincon for solving the DC PF problem for both test cases.

6.2. Simulation of a trolleybus network. In this section, we present numerical simulations of a part of a real network. The simulations are performed in the Eclipse SUMO simulator [12], [9]. We show two test cases, in both we employ our Algorithm 2 to solve the DC PF problem.

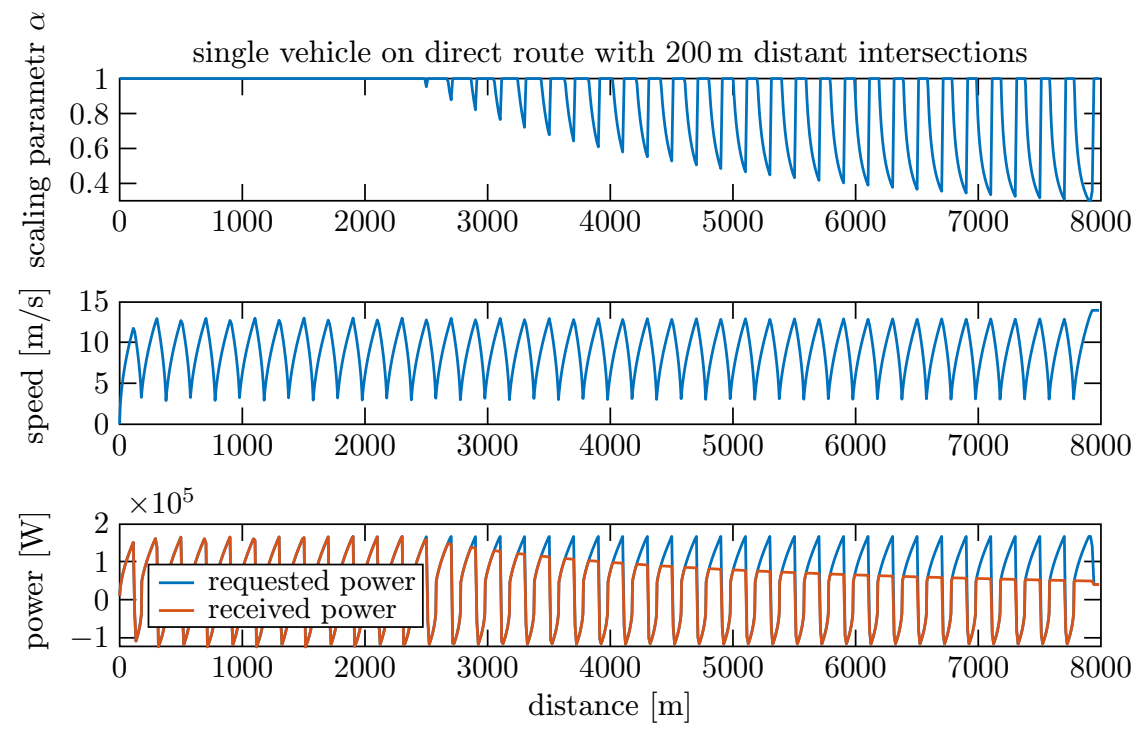

Figure 5. Simulation of single trolleybus of 8 kilometers on a straight route with equally distant $(200 \mathrm{~m})$ intersections.

The first case is a simulation of a single trolleybus on a straight 8 kilometres long route with equally distant $(200 \mathrm{~m})$ intersections. The overhead wire network is powered by a traction substation at the beginning (position $0 \mathrm{~m}$ ) of the route. Figure 5 shows the results. In the top row, the actual value of parameter $\alpha$ is depicted. The value depends on the requested power (depicted in the bottom row), which is periodically oscillating as the trolleybus accelerates after passing each intersection and uses regenerative breaking before reaching the next one. The scaling parameter decreases 
(when accelerating) with the distance from the power source due to increased length and therefore also resistance of the overhead power line. This driving behaviour with the actual speed of the trolleybus is shown in the middle row. The last row shows the requested power and the received power. The latter is the requested power times the scaling constant. Note that the difference needs to be either covered by an additional source such as an on-board battery (this is the depicted case), or it results in slower acceleration.
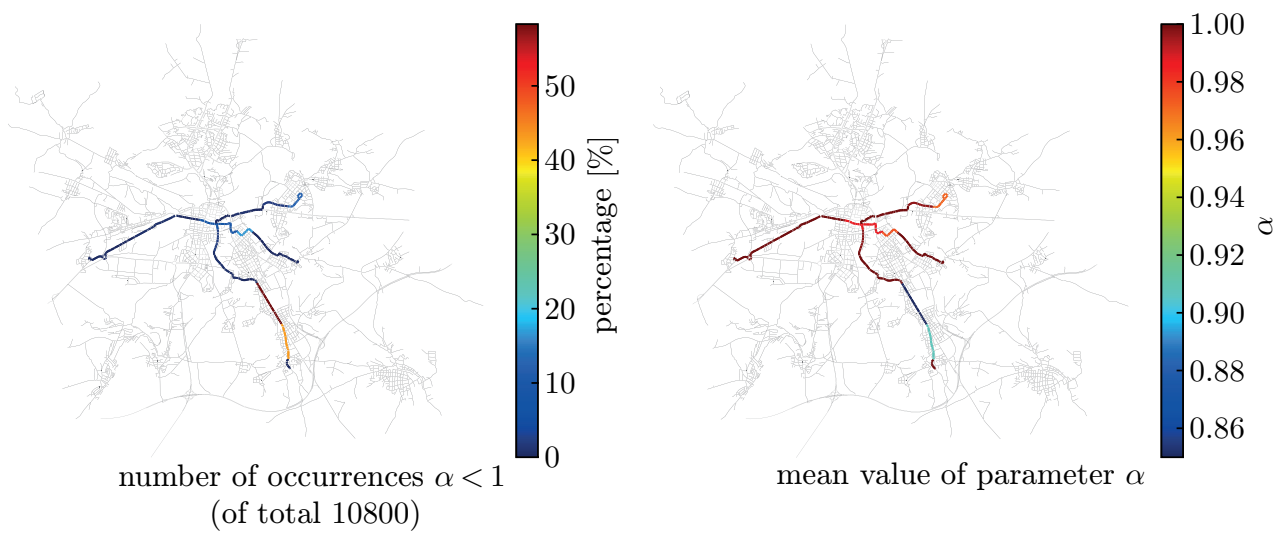

Figure 6. Large-scale stress analysis of two trolleybus lines in the city of Pilsen, Czech Republic.

The second case is a large-scale simulation of two hybrid-trolleybus lines in the city of Pilsen, Czech Republic. Figure 6 shows the frequency of application of the proposed scaling parameter in simulations (it is smaller than one) and its mean value. The results indicate sufficient dimensions of overhead wires in the city with an exception in the south side of the city. In this location, mean value of parameter $\alpha$ is under 0.9 and the relative frequency of $\alpha<1$ is above $40 \%$. This may indicate problems in the network operation and the necessity to add more substation connection points and/or overhead wire clamps into the circuit in order to improve its electrical parameters and decrease the demonstrated energy losses on the power line.

\section{Conclusion}

The formulation of the DC power flow problems leads to a system of non-linear equations that is not always solvable in its given form, despite the fact that measurements and experiments on its real-world counterpart suggest that the physical system is able to reach a state for which a real solution exists. To ensure solvability of the DC PF problem, we proposed to introduce a scaling parameter for power 
demands and presented a fast algorithm to maximise its value while keeping the DC PF system solvable, together with theoretical and numerical verification. The introduced strategy enables us to effectively find power demand thresholds and thus to solve ill-defined DC PF problems.

The algorithm has been demonstrated on representative toy cases. The performance of Matlab implementation of the proposed method has been compared to Matlab's fmincon; we demonstrated that our approach outperforms the standard optimisation approach by a factor of 6 to 9 . The viability of the algorithm and its $\mathrm{C}++$ implementation build into the open-source traffic simulator Eclipse SUMO has been demonstrated on a real-world scenario. The $\mathrm{C}++$ implementation is opensourced and is provided to the community by authors as a part of Eclipse SUMO.

\section{References}

[1] E. L. Allgower, K. Georg: Numerical Continuation Methods: An Introduction. Springer Series in Computational Mathematics 13. Springer, Berlin, 1990.

[2] H.-D. Chiang, T. Wang: Novel homotopy theory for nonlinear networks and systems and its applications to electrical grids. IEEE Trans. Control Netw. Syst. 5 (2018), 1051-1060. zbl MR doi

[3] A. L. Dontchev, R. T. Rockafellar: Implicit Functions and Solution Mappings: A View from Variational Analysis. Springer Monographs in Mathematics. Springer, New York, 2009.

[4] F. Dörfler, J. W. Simpson-Porco, F. Bullo: Electrical networks and algebraic graph theory: Models, properties, and applications. Proc. IEEE 106 (2018), 977-1005.

5] A. Garcés: Uniqueness of the power flow solutions in low voltage direct current grids. Electric Power Systems Research 151 (2017), 149-153.

[6] A. Garcés: On the convergence of Newton's method in power flow studies for DC microgrids. IEEE Trans. Power Syst. 33 (2018), 5770-5777.

[7] A. Garcés, O.-D. Montoya: A potential function for the power flow in DC microgrids: An analysis of the uniqueness and existence of the solution and convergence of the algorithms. J. Control Automation Electr. Syst. 30 (2019), 794-801.

[8] C.-W.Ho, A.Ruehli, P.Brennan: The modified nodal approach to network analysis. IEEE Trans. Circuits Syst. 22 (1975), 504-509.

[9] Institute of Transportation Systems at the German Aerospace Center: Eclipse SUMO Simulation of Urban MObility. Available at https://www.eclipse.org/sumo/ (2021).

[10] C. Jayarathna, P. Binduhewa, J. Ekanayake, J. Wu: Load flow analysis of low voltage DC networks with photovoltaic. 2014 9th International Conference on Industrial and Information Systems (ICIIS). IEEE, Piscataway, 2014, pp. 536-541.

11] B.-Y.Ku, J.-S. Liu: Solution of DC power flow for nongrounded traction systems using chain-rule reduction of ladder circuit Jacobian matrices. ASME/IEEE Joint Conference on Rail. IEEE, Piscataway, 2002, pp. 123-130.

[12] P. A. Lopez, M. Behrisch, L. Bieker-Walz, J. Erdmann, Y.-P. Flötteröd, R. Hilbrich, L.Lücken, J.Rummel, P. Wagner, E. Wiessner: Microscopic traffic simulation using SUMO. 21st IEEE International Conference on Intelligent Transportation Systems. IEEE, Piscataway, 2018, pp. 2575-2582.

[13] O. D. Montoya, L. F. Grisales-Noreña, D. González-Montoya, C. A. Ramos-Paja, A. Garcés: Linear power flow formulation for low-voltage DC power grids. Electric Power Systems Research 163 (2018), 375-381. 
[14] M.Salih, D. Baumeister, M. Wazifehdust, P. Steinbusch, M.Zdrallek, S. Mour, P. Deskovic, T. Küll, C. Troullier: Impact assessment of integrating novel battery-trolleybuses, PV units and EV charging stations in a DC trolleybus network. 2nd E-Mobility Power System Integration Symposium. KTH-Royal Institute of Technology, Stockholm, 2018, pp. 1-6.

[15] J. Sevcik, J.Prikryl: A vehicle device tailored for hybrid trolleybuses and overhead wires implementation in SUMO. SUMO User Conference 2019 (M. Weber et al., eds.). EPiC Series in Computing. EasyChair, 2019, pp. 145-157.

[16] S. Taheri, V. Kekatos: Power flow solvers for direct current networks. IEEE Trans. Smart Grid 11 (2020), 634-643.

[17] C.W.Tan, D. W. H. Cai, X. Lou: DC optimal power flow: Uniqueness and algorithms. 2012 IEEE Third International Conference on Smart Grid Communications. IEEE, Piscataway, 2012, pp. 641-646.

[18] E. Zhou, A. Nasle: Simulation of DC power distribution systems. Proceedings of Industrial and Commercial Power Systems Conference. IEEE, Piscataway, 1994, pp. 191-195.

Authors' addresses: Jakub Ševčík (corresponding author), Jan Přikryl, Václav Śmídl, University of West Bohemia, Research and Innovation Centre for Electrical Engineering, Univerzitní 2732/8, 30100 Plzeň, Czech Republic, e-mail: jsevcik@rice.zcu.cz, prikryl @rice.zcu.cz, vsmidl@rice.zcu.cz; Lukáš Adam, Czech Technical University in Prague, Faculty of Electrical Engineering, Technická 2, 16627 Praha 6, Czech Republic, e-mail: lukas. adam.cr@gmail.com. 